\section{Case Reports in Ophthalmology}

\title{
Corneal Ring Infiltrates Caused by Serratia marcescens in a Patient with Human Immunodeficiency Virus
}

\author{
Winai Chaidaroon Sumet Supalaset \\ Department of Ophthalmology, Faculty of Medicine, Chiang Mai University, \\ Chiang Mai, Thailand
}

\section{Keywords}

Serratia marcescens $\cdot$ Corneal ring infiltrates $\cdot$ Human immunodeficiency virus $\cdot$ Contact lens

\begin{abstract}
Purpose: To describe corneal ring infiltrates caused by Serratia marcescens in a patient with human immunodeficiency virus (HIV-1) who wore contact lenses. Methods: A case study of a patient with keratitis due to an infection caused by S. marcescens and exhibiting corneal ring infiltrates was reviewed for history, clinical manifestation, microscopic study, and management. Results: A 29-year-old man who had a history of contact lens wear and HIV-1 infection was admitted to hospital because of blurred vision, redness, and corneal infiltrates in the shape of a ring in the left eye. The visual acuity (VA) in both eyes was hand movement (uncorrected). Corneal scrapings were performed. The culture results of the corneal specimens revealed S. marcescens. The culture results of the contact lens disclosed the same organism. The corneal ulcer responded well to treatment with topical gentamycin sulfate $14 \mathrm{mg} / \mathrm{ml}$. The final VA remained hand movement. Conclusions: $S$. marcescens can cause ring infiltrates in a HIV-1 patient who wears contact lenses. The treatment result for $S$. marcescens keratitis in a HIV-1 patient who wore contact lenses was favorable after intensive use of fortified topical antibiotics.

(C) 2016 The Author(s)

Published by S. Karger AG, Basel
\end{abstract}




\section{Case Reports in Ophthalmology}

\section{Introduction}

Serratia marcescens keratitis is commonly found in patients who have an abnormal ocular surface and wear contact lenses. The clinical presentation is characterized by diffuse haziness of the corneal epithelium and central or peripheral infiltrates with hypopyon [1]. Corneal ring infiltrate is an uncommon manifestation of $S$. marcescens keratitis but may be found in various circumstances such as Pseudomonas, herpetic, Acanthamoeba, or fungal keratitis. We report a human immunodeficiency virus-1 (HIV-1)-infected patient who had a history of contact lens wear and developed ring infiltrate keratitis. The microscopic examination from the corneal specimen showed $S$. marcescens.

\section{Case}

A 29-year-old male presented at the cornea clinic, complaining of redness, pain, watering, and photophobia in the left eye for 3 days. He had been using disposable daily wear contact lenses for myopic correction for 2 years' duration. The patient reported that 2 days ago he slept with his contact lenses overnight and woke up with purulent discharge in his left eye. He was known to be positive for HIV-1 since 2002. His CD4 T lymphocyte count was 155 cells/ $\mu l$, currently being treated with highly active antiretroviral therapy. He had been using chloramphenicol eye drops, which he bought from a pharmacy for 1 day.

Physical examination revealed a thin man without skin lesions. Visual acuity without correction was hand movements in both eyes. The left eyelids appeared to be edematous. The conjunctiva was mixed injected. Slit-lamp examination showed a round diffuse area of subepithelial to midstromal infiltration with immune ring in the left cornea (fig. 1). An epithelial defect was also found. A small hypopyon was detected in the anterior chamber. The surrounding cornea was slightly cloudy. Corneal sensation was diminished. The right eye appeared normal. Corneal scraping was performed to obtain the corneal specimens. The contact lens, lens solutions, and case were sent for microbial analysis. The microscopic workup from corneal specimens revealed numerous Gram-negative bacilli in Gram stain (fig. 2) and the culture result was $S$. marcescens sensitive to gentamicin, vancomycin, and amikacin. The contact lens culture also showed $S$. marcescens.

The patient was advised to stop using contact lenses. The treatment was started using fortified gentamicin $(14 \mathrm{mg} / \mathrm{ml})$ eye drops to the left eye hourly. Atropine was also administered four times daily. The eye drop was tapered after his left cornea had less infiltration. The corneal ring disappeared 2 weeks after admission. The corneal lesion was finally healed with scarring.

\section{Discussion}

S. marcescens was first described by Bizio in 1823 as a cause of red discoloration of cornmeal porridge ('bleeding polenta'). It was a facultative aerobic, motile Gram-negative bacilli bacteria [1]. This organism can cause purulent conjunctivitis [2], keratitis [3], scleritis [4], and endophthalmitis $[5,6]$.

Contact lens wear is a risk factor that predisposes patients to the development of infectious keratitis. In Thailand, $11 \%$ of patients with infectious keratitis occur in contact lens wearers [7]. Overnight wear of a soft contact lens is a well-recognized risk factor for keratitis 
Case Reports in
Ophthalmology

Case Rep Ophthalmol 2016;7:359-363 $10.1159 / 000448023$

www.karger.com/cop

Chaidaroon and Supalaset: Corneal Ring Infiltrates Caused by Serratia marcescens in a Patient with Human Immunodeficiency Virus

[8]. S. marcescens is commonly found in the damp environment such as the bathroom where our patient kept the contact lens [9]. A severe degree of cellular immunodeficiency is found in patients infected with HIV. This patient was HIV seropositive and had a history of sleeping with contact lenses. His decreased immune status with contact lens misuse had made him prone to the development of Serratia keratitis. HIV patients have also been associated with abnormal ocular surface abnormality and dry eye; therefore, they may be predisposed to ocular Serratia infection.

The corneal lesion seen in this patient was a stromal infiltration with an immune ring. This ring infiltrate, presumably the result of an immunologic response such as antigenantibody complexes, complement, and polymorphonuclear leukocytes, can be seen in patients with severe corneal burns, corneal foreign bodies, Pseudomonas, herpetic, Acanthamoeba, or fungal keratitis, and abuse of topical anesthetic agents. Nevertheless, we demonstrated that this immune ring is consistent with another presentation of Serratia keratitis in the contact lens-wearing patient who had seropositive HIV. Their appearance in this patient may have been modified by partial treatment or by his immunodeficiency status. However, the appearance of the corneal ring improved after treatment for 2 weeks.

S. marcescens is known to be a multidrug-resistant pathogen [6]. Fortunately, this patient had a successful treatment with fortified gentamicin eye drops.

\section{Conclusion}

Our findings showed that $S$. marcescens keratitis can present with ring infiltrate in a patient who wears contact lenses and suffers from HIV-1 infection. The treatment result was favorable with fortified gentamicin eye drops.

\section{Acknowledgements}

The authors gratefully acknowledge Mrs. Sumalee Pruksakorn, PhD, for providing the photograph of the microbiologic staining.

\section{Statement of Ethics}

The authors have no ethical conflicts to disclose.

\section{Disclosure Statement}

None of the authors has any conflicts of interest concerning this case report.

\section{References}

1 Parment PA: The role of Serratia marcescens in soft contact lens associated ocular infection. Acta Ophthalmol Scand 1997;75:67-71.

2 Atlee WE, Burns RP, Oden M: Serratia marcescens keratoconjunctivitis. Am J Ophthalmol 1970;70:3133. 
-3 Chen KH, Kuang TM, Hsu WM. Serratia marcescens corneal ulcer as a complication of orthokeratology. Am J Ophthalmol 2001;132:257-258.

4 Hwang YS, Chen YF, Lai CC, Chen HS, Hsiao CH: Infectious scleritis after use of immunomodulators. Arch Ophthalmol 2002;120:1093-1094.

-5 Mah-Sadorra JH, Najjar DM, Rapuano CJ, Laibson PR, Cohen EJ: Serratia corneal ulcer: a retrospective clinical study. Cornea 2005;24:793-800.

-6 Equi RA, Green WR: Endogenous endophthalmitis with dark hypopyon: a case report and review. Surv Ophthalmol 2001;46:259-268.

7 Chaidaroon W, Wattananikorn S: Contact lens-associated infectious keratitis in Thailand. Asian J Ophthalmol 2007;9:209-212.

8 Poggio EC, Glynn RJ, Schein OD, Seddon JM, Shannon MJ, Scardino VA, Kenyon KR: The incidence of ulcerative keratitis among users of daily-wear and extended-wear soft contact lens lenses. N Engl J Med 1989;321:779-783.

-9 Hejazi A, Falkiner FR: Serratia marcescens. J Med Microbiol 1997;46:903-912.

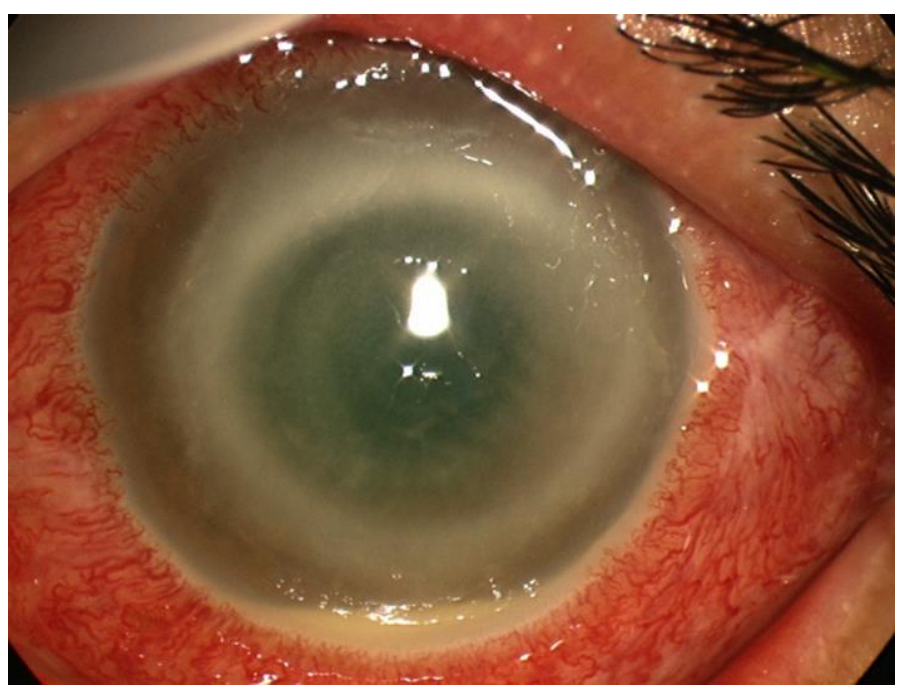

Fig. 1. Left eye of a HIV patient showing stromal corneal infiltration with active corneal ring. 
Case Reports in
Ophthalmology

Case Rep Ophthalmol 2016;7:359-363

(c) 2016 The Author(s). Published by S. Karger AG, Basel www.karger.com/cop

Chaidaroon and Supalaset: Corneal Ring Infiltrates Caused by Serratia marcescens in a Patient with Human Immunodeficiency Virus

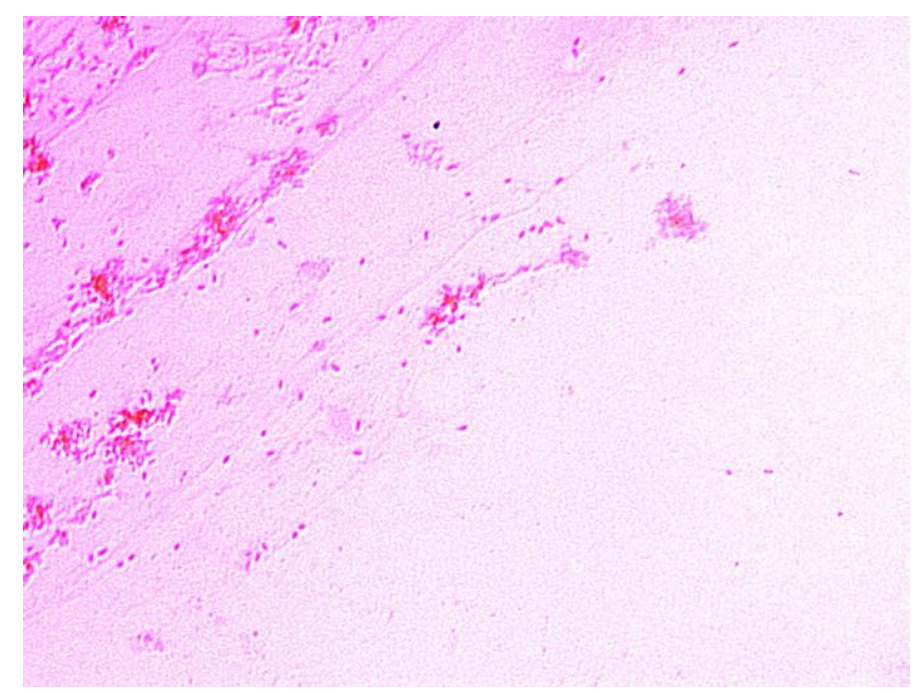

Fig. 2. Gram stain demonstrated numerous gram-negative bacilli. 